\title{
Changes in PGC-1a and Mfn1/Drp1 levels are indicative of amyloid b-induced mitocondrial dysfunction induced by P2X2-mediated Ca2+ overload in a cellular model of Alzheimer's disease
}

\author{
Jorge Fuentealba, Jessica D Panes, Tiare Silva-Grecchi, Javiera Gavian, Daniela Mennickent, \\ Pamela A Godoy, Gustavo Moraga-Cid, Gonzalo E Yevenes, Leonrardo Guzman, Patricio A Castro \\ Physiology, University of Concepcion, Chile
}

Soluble oligomers of $\mathrm{Ab}(\mathrm{SOAb})$ are the toxic agent in the pathogenesis of $\mathrm{AD}$ with multiple toxic effects. The aim of this work was to evaluate the effects of SOAb on the expression and levels of P2X2R and its contribution on mitochondrial biogenesis and dynamics. Changes on P2X2R was measured and Mitochondrial biogenesis was evaluated by changes on peroxisome proliferator-activated receptor gamma coactivator 1-alpha (PGC-1a), and by the silent mating type information regulation 2 homolog (SIRT1) protein. On the other hand, mitochondrial dynamics was evaluated by changes on the fusion protein Mitofusin 1 (Mfn1) and fission dynamin-related protein 1 (DRP1). P2X2R increase (after $24 \mathrm{~h}$ ) its expression and functional activity on neurons (over 25\%). Through western blot and immunofluorescence, we observed decreased SIRT1 after SOAb treatments (about 50\%). Conversely, an increment of PGC-1a was detected after 5h incubations $(218 \%)$, though reduced levels were obtained after 1 and $24 \mathrm{~h}$ treatments $(30 \%, 42 \%$ respectively). The interaction between these two proteins after a $24 \mathrm{~h}$ SOAb exposure was lost, which correlates with a change in PGC-1a distribution, increasing in the cytoplasm (177\%) and decreasing in the nucleus (38\%). After $2 \mathrm{~h}$ incubations we observed a reduction in Mfn1 levels (42\%), and an increase in DRP1 (198\%); additionally, DRP1 presented a distribution shift on hippocampal neurons, increasing its immunoreactivity in neuronal processes. Changes regarding mitochondrial proteins correlate with an alteration of mitochondrial network morphology and this dysfunction can be associated to the P2X2R contribution. Finally, SOAb-induced imbalance on P2X2R, mitochondrial dynamics, and decreased mitochondrial biogenesis, thus blocking the pathway that lead to the cell to have functional mitochondrias. 